\title{
A New Perspective for Relating Virtual Inertia with Wideband Oscillation of Voltage in Low-Inertia DC Microgrid
}

\section{Li, Chang; Yang, Yaqian; Dragicevic, Tomislav; Blaabjerg, Frede}

Published in:

IEEE Transactions on Industrial Electronics

Link to article, DOI:

10.1109/TIE.2021.3100932

Publication date:

2022

Document Version

Peer reviewed version

Link back to DTU Orbit

Citation (APA):

Li, C., Yang, Y., Dragicevic, T., \& Blaabjerg, F. (2022). A New Perspective for Relating Virtual Inertia with Wideband Oscillation of Voltage in Low-Inertia DC Microgrid. IEEE Transactions on Industrial Electronics, 69(7), 7029-7039. https://doi.org/10.1109/TIE.2021.3100932

\section{General rights}

Copyright and moral rights for the publications made accessible in the public portal are retained by the authors and/or other copyright owners and it is a condition of accessing publications that users recognise and abide by the legal requirements associated with these rights.

- Users may download and print one copy of any publication from the public portal for the purpose of private study or research.

- You may not further distribute the material or use it for any profit-making activity or commercial gain

- You may freely distribute the URL identifying the publication in the public portal 


\title{
A New Perspective for Relating Virtual Inertia with Wideband Oscillation of Voltage in Low-Inertia DC Microgrid
}

\author{
Chang Li, Yaqian Yang, Tomislav Dragicevic, Senior Member, IEEE, Frede Blaabjerg, Fellow, IEEE
}

\begin{abstract}
Virtual synchronous generator (VSG) has been a grid-friendly integration control technique for the integration of grid-connected inverters. However, the emulated inertia and damping of VSG control technique can also be used in the field of DC systems. In this paper, a virtual synchronous control is proposed to dampen the wideband oscillation of DC voltage in a DC microgrid. The proposed control strategy contributes to maintaining synchronous operation of DC converter with the network. Besides, the relationships among damping, inertia, wideband oscillation, rate of change of voltage $(\mathrm{RoCoV})$ as well as DC voltage nadir (DCVN) are studied. It is concluded that the RoCoV and DCVN are similarly as the oscillation frequency and fluctuation ranges of poorly-damped oscillation, respectively. A unified concept is proposed by connecting the oscillation-related stability with inertial transient response originated from the imbalanced powers/mismatched currents. Besides of this, the inertia plays the same role as damping because the inertia contributes to maintaining the original state and damping to impeding further change. A new feedback analytical method is proposed to clarify the important role of RoCoV and DCVN on the motion of DC voltage. Finally, the theoretical results are compared with simulations and experiments.
\end{abstract}

Index Terms-rate of change of voltage ( $\mathrm{RoCoV})$, DC voltage nadir (DCVN), inertia and damping, virtual synchronous generator (VSG), poorly-damped oscillations, feedback analytical method

\section{INTRODUCTION}

$\mathrm{E}^{\mathrm{N}}$ NVIRONMENTAL pollution and energy crisis have been troublesome issue, which needs to be tackled urgently. Aroused by this stimulation, large-scale renewable energy sources, energy-storage system and electric vehicles have been interfaced with the grid via power electronic converters to alleviate the burden of conventional fossil fuels [1]. Unfortunately undesirable interactive behaviors between power converter and grid may induce wideband oscillations due to the poor damping [2]. At the same time fast response from the converter can result in low-inertia and weak-damping, which can jeopardize the stable operation of the system [3].

Power converters with low-inertia and weak-damping can

Manuscript received on $3^{\text {rd }}$ December, 2020, and revised on $17^{\text {th }}$ February, $2021,19^{\text {th }}$ April, 2021, and $17^{\text {th }}$ June, 2021, accepted on $17^{\text {th }}$ July, 2021

Chang $\mathrm{Li}$, and Tomislav Dragicevic are with Technical University of Denmark, Denmark. (corresponding author: Chang Li) E-mail: changlielektro@gmail.com

Yaqian Yang is with Hunan University, China.

Frede Blaabjerg is with Aalborg University, Denmark. interact with the DC network, resulting in undesirable interactions or even instability of the systems [4]. The leading cause has been found in the form of a resonance loop at high frequency bands between two subsystems impedance. Similarly, the mechanism of the oscillation phenomenon has been illustrated by eigenvalue analysis, which found poorly-damped dominant oscillatory modes in DC microgrid [6]. In addition, a feedback analytical method was proposed to identify whether divergent oscillation or convergent oscillation of the voltage in a DC system [15]. In summary, the leading cause of voltage oscillation in DC microgrid can be summarized as three aspects: 1) undesirable impedance interaction between converter and the DC network, 2) poorly-damped critical modes, and 3) positive feedback leading to divergent oscillation.

To overcome those instability issues, virtual impedance (VI) [4]-[6] and virtual synchronous machine (VSM) [7]-[13] are the two main categories used to provide support for inertia and damping for the power system. Compared to VI control method, VSM control has certain advantages, e.g. provision of inertial support, relatively easier implementation, etc. VSM can be divided into two categories including AC VSM and DC VSM. AC VSM is generally used to improve the frequency stability and transient response during the disturbance [10]-[13]. DC VSM is usually used for inertia and damping support of DC voltage [14]-[17]. In addition, several papers have also investigated the VSM and virtual inertia, including parameter constraints [18], small signal modeling [19], [20] stability assessment [21], and the inertia design [22].

In some references, a virtual inertia control is used for improving the transient response of the system, i.e., alleviating the RoCoF and FN [23]-[25] or rate of change of voltage (RoCoV) or DC voltage nadir (DCVN) [16], [17]. No matter of RoCoF, FN, RoCoV, or DCVN, it is attributed to the imbalanced active powers which compels the frequency or DC voltage deviate from the nominal value, and thus a RoCoF (RoCoV) and FN (VN) has been emerging.

In fact, the main focus of inertia analysis is generally concentrated on the transient nadir and the rate of change of the state variables. However, for the topics of oscillation-related stability (ORS), the voltage oscillation amplitude (VOA) and oscillation frequency (OF) are usually the main focus. It can be seen in Fig. 1 that the transient nadir has the highly similarity with the oscillation amplitude, and the rate of change of voltage can be analogous to oscillation frequency of the voltage.

As shown in Fig. 1(a), a power disturbance occurs and thus 
the transient deviation of DC voltage appears. Besides, an attenuated oscillation occurs due to the weak damping of the system, as shown in Fig. 1(b). In fact, the expression of attenuated oscillation can be derived as a standard second-order form:



(a)

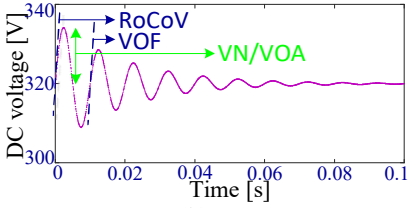

(b)
Fig. 1. Analogy between inertia analysis and oscillation-based stability analysis, (a) transient response during imbalanced power, (b) attenuated oscillation with weak damping.

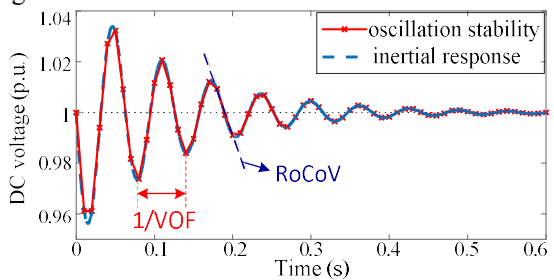

(a)

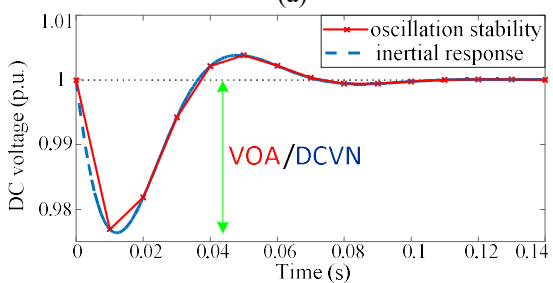

(b)

Fig. 2. Comparison between ORS and IR with RoCoV and VOF, DCVN and VOA, (a) smaller damping, (b) larger damping.

Perturbing the above (1) at $\mathrm{t}=0 \mathrm{~s}$, the slope of the curve at $\mathrm{t}=0$ s means maximum RoCoV, i.e.,

$$
\begin{aligned}
& f^{\prime}(t)=-\xi \omega_{n} e^{-\xi \omega_{n} t} \sin \left(\omega_{d} t\right)+\omega_{d} e^{-\xi \omega_{n} t} \cos \left(\omega_{d} t\right) \\
& \Rightarrow f^{\prime}(t=0)=\omega_{d}
\end{aligned}
$$

From (2), it can be inferred that the maximum of RoCoV from the perspective of inertia analysis is equal to the VOF from the view of ORS analysis. Furthermore, the voltage nadir arises at $\mathrm{RoCoV}=0$, i.e., slope of curve $=0$, within the first cycle.

The step response is a good way to identify the IR with consideration of RoCoV and DCVN when system is subjected to a disturbance of imbalanced powers. Thus, the second-order model of the attenuated oscillation can be derived as:

$$
H_{\text {step }}(s)=\frac{\omega_{d}}{\left(s+\xi \omega_{n}\right)^{2}+\omega_{d}{ }^{2}}
$$

where $H_{\text {step }}(s)$ can be used to identify the performance of transient deviations, i.e., RoCoV, and DCVN.

It can be seen from Fig. 2 that the RoCoV is justly equal to VOF, and the DCVN is justly as the same as VOA. In addition, the inertial response (IR), which is originated from mismatched powers, is as the same form as the oscillation-related stability due to weak damping and undesirable interactions, which can be shown in Fig. 2. It can be inferred that the IR of the DC voltage cause by the imbalanced powers has the similar characteristics with that of DC voltage oscillation due to the undesirable interactions instability.

To make clearer concepts for the classifications among
RoCoF, RoCoV, FN, DCVN, VOA, and VOF, the difference between IR (subjected to imbalanced powers) and ORS (during small-perturbations) are identified by Table I.

In Table I, MFD represents maximum frequency deviations, MVD stands for maximum voltage deviations, IP denotes imbalanced powers, and RSP means random small perturbation.

TABLE I. CLASSIFICATION ACCORDING TO IR AND ORS

\begin{tabular}{c|c|c|c|c|c|c}
\hline \multicolumn{1}{c}{ Items } & \multicolumn{4}{c|}{ IR } & \multicolumn{2}{c}{ ORS } \\
\hline $\begin{array}{c}\text { Index } \\
\text { classification }\end{array}$ & RoCoF & RoCoV & FN & DCVN & VOA & VOF \\
\hline Literatures & $\begin{array}{c}{[3]} \\
{[7]-[13]} \\
{[18]-[25]}\end{array}$ & [14]-[17] & $\begin{array}{r}{[3][7]} \\
-[13] \\
{[18]-} \\
{[25]}\end{array}$ & $\begin{array}{c}{[14][15]} \\
{[16][17]}\end{array}$ & $\begin{array}{c}{[2][4][6]} \\
{[14]} \\
{[15]}\end{array}$ & $\begin{array}{c}{[2][4][6][} \\
14] \\
{[15]}\end{array}$ \\
\hline $\begin{array}{c}\text { Dynamic } \\
\text { characteristic }\end{array}$ & RoCoF & RoCoV & MFD & MVD & MVD & RoCoV \\
\hline $\begin{array}{c}\text { Disturbance } \\
\text { source }\end{array}$ & IP & IP & IP & IP & RSP & RSP \\
\hline
\end{tabular}

From the view of physical significance, inertia contributes to compelling the state variables to maintain the original state and thus not being changed. However, the effect of damping aims to impede the state variables further deviating from the nominal value and compel it convergent to the given value.

Based on this idea, it has been pointed out that transient voltage nadir has the similar characteristics with that of oscillation of DC voltage. Thus, the proposed virtual synchronous control can implement the "synchronized operation" of DC voltage. The main contributed works are organized as follows:

1) The principle of synchronization of DC converter with the grid is clarified. And the impact of inertia and damping on the synchronization of DC voltage is explored. A new perspective of voltage oscillation mechanism is clarified that voltage oscillation is originated from the periodic fluctuation of DC current and periodically charging or discharging current through DC capacitors.

2) This paper put forward the unified concept for relating the RoCoV and DCVN with the interaction-related oscillation for the first time. And it builds the connection between RoCoV and oscillation frequency, and it also builds up the relationship between DCVN and oscillation magnitude. Besides, the impact of virtual inertia and virtual damping on RoCoV, DCVN, VOF, and VOA are discussed comprehensively.

3) It is found that larger size of virtual inertia can bring better damping performance. Furthermore, the larger inertia and stronger damping can make the better synchronization ability of the DC voltage with the DC microgrid.

4) The dynamic interaction between RoCoV and DCVN is clarified by the proposed feedback effect concept. Both of RoCoV and DCVN play important roles in the motion of the voltage.

5) The feedback analytical method and principle are initially proposed to illustrate the motion of voltage in a DC microgrid, with relating RoCoV and DCVN with the ORS. A unified concept is initially proposed by relating RoCoV with VOF, and relating DCVN with VOA.

The rest of this paper is organized as follows: Section II briefly introduces the mechanism of periodical fluctuation of DC voltage. Section III discusses the dynamic interaction 
between RoCoV and DCVN, besides, the unified concept of relating RoCoV and DCVN with ORS is proposed. In Section IV, theoretical analysis is verified by simulations and

\section{Small Signal Model of Virtual Synchronous CONTROL}

In this section, the mechanism of voltage fluctuation is firstly illustrated. Then, a virtual synchronous control is proposed to implement the synchronized operation of DC voltage with the help of enough inertia and damping.

\section{A. Brief introduction of instability of mechanism}

Fig. 3 shows the topology of a DC microgrid. In reality, the oscillating DC current can result in the fluctuation of DC voltage around the nominal point through the charge and discharge of DC capacitor. Hence, the dynamics of DC current loop can motivate the oscillation of DC voltage. Fig. 4(a) shows the DC current closed-loop, and Fig. 4(b) is the frequency response of the transfer function.

It can be seen in Fig. 4(b), that there exist resonance peaks with various DC grid impedances with a small phase margins. Thus, the current is prone to oscillation which results in the periodical fluctuation of DC voltage.

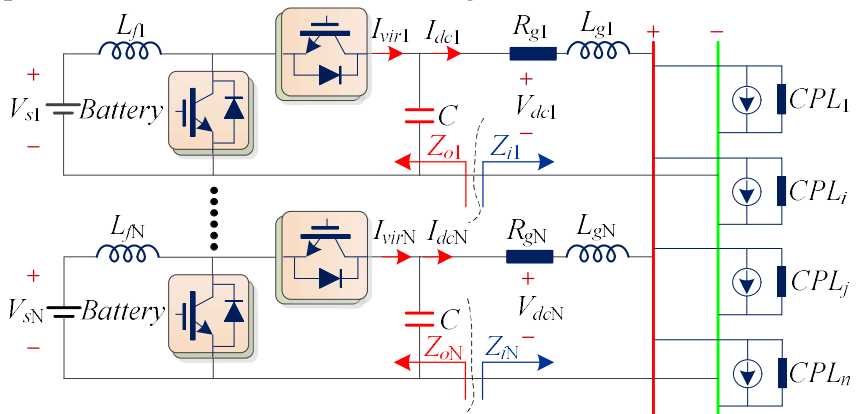

Fig. 3. The topology of DC microgrid with energy storage.

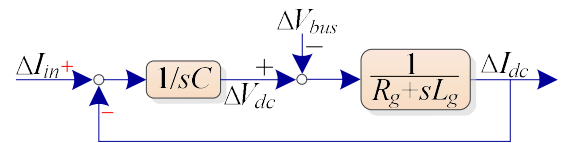

(a)

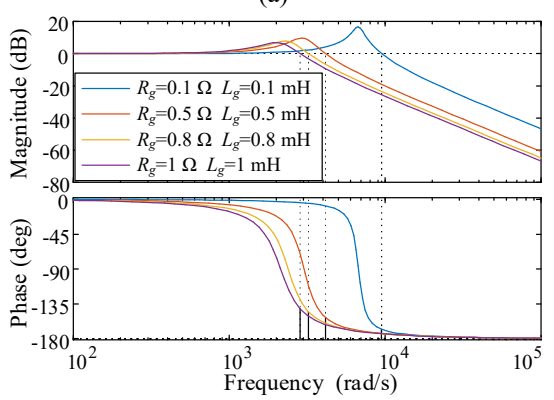

(b)

Fig. 4. DC current control loop, (a) Closed-loop control diagram, (b) frequency responses

\section{B. Modeling of virtual synchronous control}

Fig. 5(a) displays the control diagram of DC/DC converter with virtual synchronous control. The swing equation of virtual synchronous control, which emulates the behavior of inertia and damping can be derived, can be expressed as: experiments. Finally, Section V gives the conclusions and discussions.

$$
\frac{1}{J_{v i r}} \omega_{N} \frac{d \omega_{v i r}}{d t}=P_{m}-P_{d c}-k_{d a m} \omega_{N}\left(\omega_{v i r}-\omega_{N}\right)
$$

where $k_{d a m}=k+D_{d a m}$, which incorporate both damping effect and droop effect. $\omega_{N}$ is an adjustable parameter which can be flexibly set. However, here it is set to $50 \mathrm{~Hz}(314 \mathrm{rad} / \mathrm{s})$ to emulate the characteristics of synchronous generator. To provide inertia support for DC voltage, the virtual angular frequency $\omega_{v i r}$ is coupled with virtual DC voltage through a proportional gain $f_{y T}$, i.e.,

$$
V_{v i r}=\omega_{v i r} f_{y T}
$$

where $V_{\text {vir }}$ is the obtained virtual DC voltage, the virtual DC current is further derived by the following equation:

$$
I_{v i r}=\frac{1}{k_{v i r t}}\left(V_{v i r}+V_{d c}\right)
$$

where the feedforward term $V_{v i r}$ can provide inertia and damping support for DC system by the emulated virtual inertia and virtual damping. Besides, $k_{\text {virt }}$ is a supplementary damping term to provide damping support. It should be noted that (4)-(6) depict the dynamic behavior of virtual synchronous control, and the virtual synchronous control loop is cascaded between outer voltage control loop and current inner loop, which can be shown in Fig. 5(a).

Perturbing (4)-(6), the small signal model can be derived as:

$$
\left\{\begin{array}{c}
\frac{1}{J_{v i r}} \omega_{N} \frac{d \Delta \omega_{v i r}}{d t}=\Delta P_{m}-\Delta P_{d c}-k_{d a m} \omega_{N}\left(\Delta \omega_{v i r}-\Delta \omega_{N}\right) \\
\Delta V_{v i r}=f_{y T} \Delta \omega_{v i r} \\
\Delta I_{v i r}=\frac{1}{k_{v i r t}}\left(\Delta V_{v i r}+\Delta V_{d c}\right)
\end{array}\right.
$$

where it can be seen that both variation of DC voltage and virtual DC voltage can produce the virtual DC current to provide inertial support to assist the synchronized operation of DC voltage, besides, it should be noted that the energy source to provide inertia and damping support incorporate two components, i.e., $\Delta P_{m}$ and $\Delta P_{\text {dam }}$.

Specifically, $\Delta P_{m}$ is generated by the output of regulation of outer voltage control loop, and the output is reliable on the variation of DC voltage. That is to say, the much faster variation rate and larger fluctuation range can generate more virtual input power for support of inertia and damping. Moreover, the damping power contributes to impeding the further change of DC voltage, which can make DC voltage attenuated much faster. Meanwhile, the larger $k_{\text {dam }}$ leads to less dynamic deviation of DC voltage and voltage nadir, and larger $k_{\text {dam }}$ produces more damping powers to support inertia.

Indeed, the swing equation of the virtual synchronous control behaves as a low-pass filter, i.e.,

$$
H_{v s}=\frac{1}{k_{\text {dam }}} \frac{1}{\frac{s}{J_{v i r} k_{d a m}}+1}
$$

where $H_{v s}$ behaves like a first-order inertial link, and $J_{v i r}$ and $k_{\text {dam }}$ have the same effect to contribute to the inertia support, 


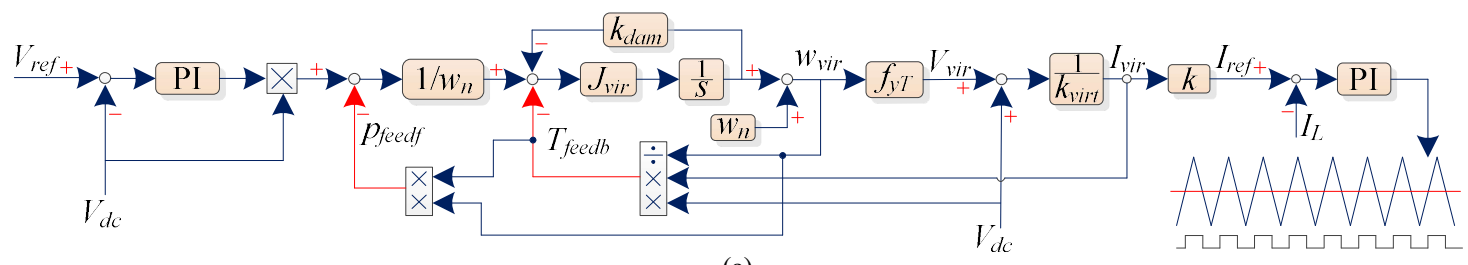

(a)

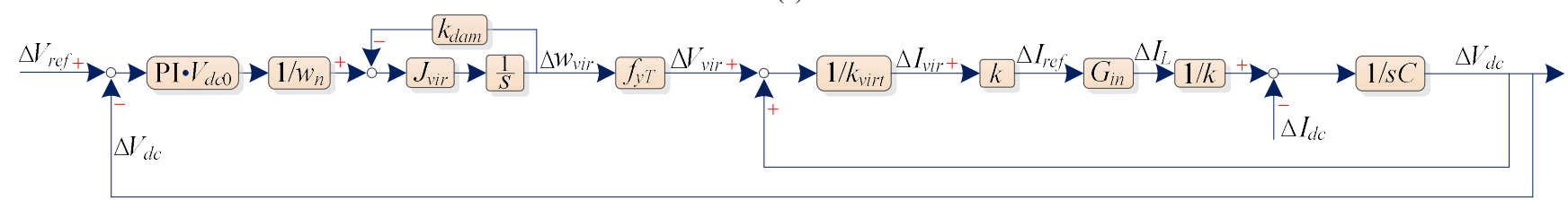

(b)

Fig. 5. Virtual synchronous control, (a) architecture of virtual synchronous control, (b) small signal model of DC voltage closed-loop with virtual synchronous control

which also verifies that the virtual inertia and virtual damping have the same effect on improving the transient response.

From physical view, larger inertia means that the DC voltage can better maintain the original state; meanwhile, the larger damping suggests that the DC voltage is impeded further changed more. Thus, the inertia has the same effect as the damping in this control structure.

Moreover, larger $J_{v i r}$ and $k_{d a m}$ means the faster response of the active power to provide more inertia and damping support for DC voltage during the transient responses.

Fig. 6 displays the effect of inertia and damping on the dynamic response of DC voltage by means of test of step response. It can be seen in Fig. 6(a) that the larger size of virtual inertia has several advantages, e.g., smaller fluctuation ranges, less DC voltage nadir, less settling time, and more robust transient response. Furthermore, it can be inferred that transient response caused by imbalanced powers is essentially has the same dynamics as the oscillation-related dynamics because it has been clarified that the periodical fluctuation of the current through DC capacitors is the main cause of periodical charging and discharging of DC capacitors.

It illustrates that larger virtual inertia can reduce the dynamic deviation of DC voltage. In addition, it can compel DC voltage to arrive at the steady state much faster with less oscillation. Fig. 6(b) shows that larger virtual damping factor owns several advantages, e.g., less settling time, less fluctuation ranges, less voltage nadir, and more stable DC voltage. When $k_{\text {dam }}$ arrives at 5 , there exists no any oscillation and DC voltage can quickly restore to steady state.

It can be inferred that both virtual inertia and virtual damping contributes to compelling DC voltage maintain the original state and impeding it further changed. Thus, DC voltage can be divergent and stable with less time and less fluctuation.

\section{STABILITY AND DYNAMICS ANALYSIS}

In this Section, the dynamic interaction between RoCoV and DCVN is discussed by the proposed feedback analysis. Besides, a unified concept of relating virtual inertia with ORS is illustrated with feedback effect.

\section{A. Nyquist criterion based on minor-loop gain}

The Nyquist criterion based on minor-loop gain proposed by Middlebrook is an effective way to identify the interaction between two subsystems in a cascaded network [26]. Based on the laws of Kirchhoff and energy conservation, perturbing the circuit equation around the equilibrium point, one can obtain:

$$
\left\{\begin{array}{c}
\Delta V_{s i}-\Delta E_{i}=L_{f i} \frac{d \Delta I_{L i}}{d t} \\
\Delta V_{s i} I_{L i 0}+V_{s i 0} \Delta I_{L i}=\Delta V_{d c} I_{d c 0}+V_{d c 0} \Delta I_{d c}+C V_{d c 0} \frac{d \Delta V_{d c}}{d t}
\end{array}\right.
$$

where $V_{s i}$ and $E_{i}$ represent source voltage and inner potential of DC converter, $V_{d c}$ is the DC voltage across the capacitor, and $I_{d c}$ denotes the output DC current of the converter.

The control dynamic equations of virtual synchronous control are deduced as:

$$
\left\{\begin{array}{c}
\left(\Delta V_{r e f}-\Delta V_{d c}\right) \frac{k_{p v} s+k_{i v}}{s} V_{d c 0}=\Delta P_{m} \\
\Delta E=\left(\Delta I_{v i r} k-\Delta I_{L}\right) \frac{k_{p} s+k_{i}}{s}
\end{array}\right.
$$

Combining (7), (9), with (10), the output impedance of the converter with virtual synchronous control can be obtained as:

$$
Z_{o}=-\frac{\Delta V_{d c}}{\Delta I_{d c}}
$$

where $Z_{o}$ is the output impedance of the converter with the virtual synchronous control, besides, the input impedance includes the DC grid impedance as well as constant power load (CPL), i.e.,

$$
Z_{i}=R_{g}+s L_{g}+R_{L}
$$

where it can be seen $Z_{i}$ is contained in the closed loop control of DC voltage. With the minor-loop gain [26], the Nyquist criterion is developed to evaluate the interactive behavior in a cascaded system, i.e., $T_{\text {loop }}=Z_{o} / Z_{i}$.

Fig. 7 illustrates the results of Nyquist stability analysis, where the studied control system is the minor-loop gain, i.e., $Z_{i}$ divided by $Z_{o}$. Moreover, Fig. 7(a) shows the Nyquist curve without virtual synchronous control. It should be noted that the signs mean the length of DC lines. It can be seen that all of the curves encircled the point $(-1,0)$ with various length of DC line, indicating the system is unstable. Thus, conventional dual-loop control has a high risk of instability due to the unexpected impedance ratio. It should be noted that the direction of arrows means the direction of larger size of the studied parameters.

Fig. 7(b) shows the impact of virtual inertia factor on the 
stability of the system. In fact, stability margin is better as $J_{v i r}$ gets larger. The similar effect can be seen in the variation of $k_{\text {dam }}$ and $k_{\text {virt }}$. It shows a superior performance of the control algorithm. Besides of this, the control has a wide control parameters ranges that can guarantee the stability of the system.

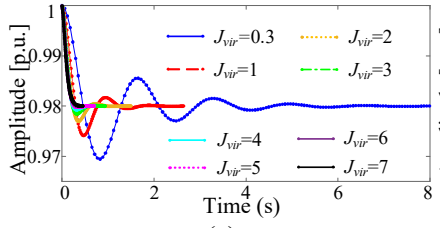

(a)

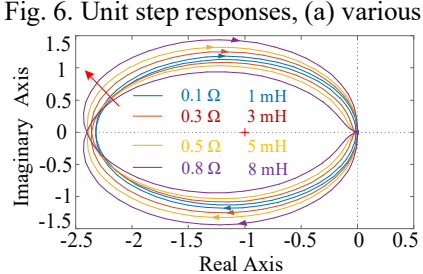

(a)

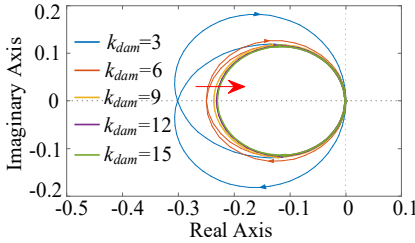

(c)

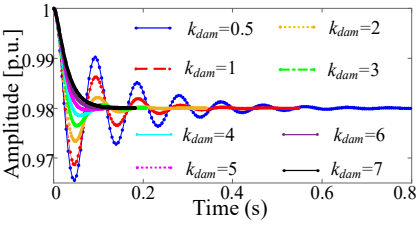

(b)

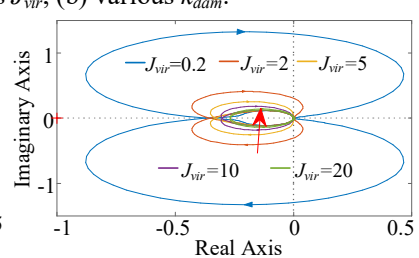

(b)

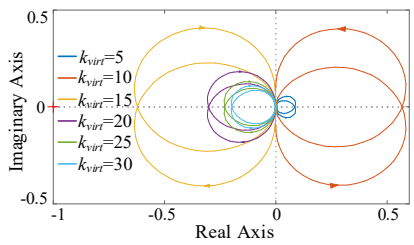

(d)
Fig. 7. Nyquist stability analysis, (a) without virtual synchronous control, (b) various $J_{v i r}$, (c) various $k_{d a m},(\mathrm{~d})$ various $k_{\text {virt }}$.

\section{B. Eigenvalue analysis}

Fig. 8(a) depicts the impact of $J_{\text {vir }}$ on the dominant poles of the system, and it shows that the oscillation frequency firstly increases and then declines as $J_{v i r}$ gets larger. Besides, the damping of the system is successively increased as $J_{v i r}$ gets larger. The phenomenon agrees well with the unit step response in Fig. 6(a). The unit step response indicates that the oscillated frequency gets higher and then becomes lower (finally equal to 0 ), and the damping is continually enlarged due to the oscillation range gets smaller.

Fig. 8(b) and (c) shows the impact of $k_{d a m}$ and $k_{\text {virt }}$ on the stability of the system. The damping of the system gets larger as the parameter becomes larger, which indicates that the larger $k_{\text {virt }}$ and $k_{\text {dam }}$ contributes to stronger damping for the system. Thus, larger sizes of $J_{v i r}, k_{v i r t}$ and $k_{d a m}$ are all good to the stability of the microgrid. It also suggests that the proposed control has a good robustness performance as well as wide operation range.

C. Dynamic interactions between RoCoV and DCVN with feedback effect

In this part, the dynamic interaction between $\mathrm{RoCoV}$ and DCVN is discussed, and those two indices in imbalanced transient response are related with the two indices, e.g., VOF, VOA, of ORS to clarify the motion of DC voltage.

Remark 1: dynamic deviation of DC voltage is defined as $\left(V_{d c}-V_{r e f}\right)$, and its maximum deviation is defined as DC voltage nadir (DCVN), which has been mentioned in the introduction. The RoCoV can be used for describing how fast the voltage changes, i.e., defined as $d V_{d c} / d t$.

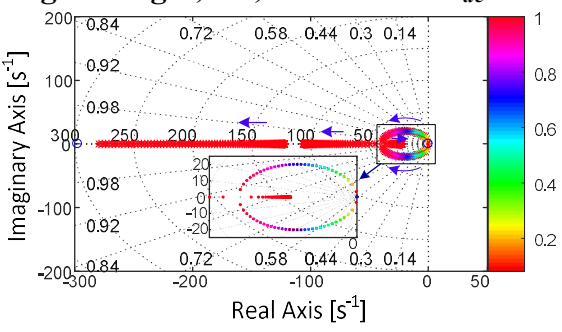

(a)

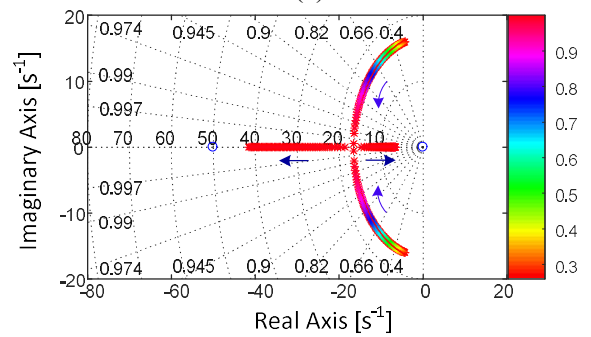

(b)

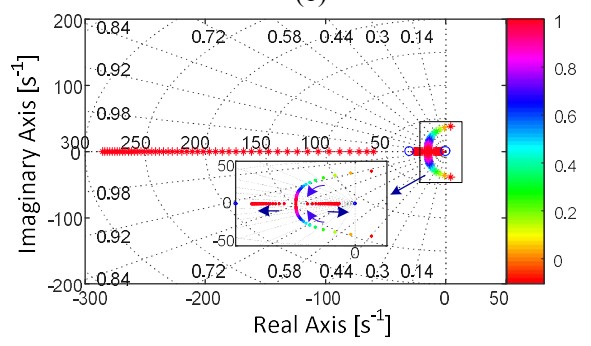

(c)

Fig. 8. Eigenvalue analysis with virtual synchronous controller, (a) $0.2 \leqslant J_{v i r} \leqslant$ 100 with a step of 1 , (b) $2 \leqslant k_{\text {dam }} \leqslant 10$ with a step of 0.05 , (c) $6 \leqslant k_{\text {virt }} \leqslant 30$ with a step of 0.5 .

Remark 2: as similar as the angular frequency of $\mathrm{AC}$ system, the dynamic deviation of DC voltage, i.e., $V_{d c}-V_{r e f}$, can be recognized as the "relative velocity" because the system is stable when $V_{d c}=V_{r e f}$ in steady state. Therefore, the RoCoV, i.e., $d V_{d c} d t$ can be seen as the "acceleration" of the system because of $d V_{d c} / d t=d\left(V_{d c}-V_{r e f}\right) / d t$ ( $V_{r e f}$ is a fixed constant).

RoCoV and DCVN are the two most important indices of DC systems. It not only can reflect the dynamic performance of the DC systems, but also can be used for the stabilization evaluation in this paper.

Discussion 1: In this discussion, the dynamic interaction between RoCoV and DCVN are clarified in one oscillation period. For example, as shown in Fig. 9(a), one oscillation period from the instant $t_{0}$ to the instant $t_{4}$ is selected as the cycle. Between the instant $t_{0}$ and instant $t_{1}$, the $\operatorname{RoCoV}$ is above zero owing to the positive slope, and the dynamic deviation $\left(V_{d c}-V_{r e f}\right)$ is always positive. It suggests that acceleration is always as the same orientation as the velocity, which compel dynamic deviation of DC voltage be enlarged continuously. In this case, it forms a positive feedback effect. Thus, it cannot be stabilized at an equilibrium point. Seen from the phenomenon, dynamic response during from $t_{0}$ to $t_{1}$ is deviating from the nominal equilibrium point, which demonstrates the discussion.

From the instant $t_{1}$ to instant $t_{2}, \operatorname{RoCoV}$ is negative, but 
dynamic deviation $\left(V_{d c}-V_{r e f}\right)$ is still always positive. It can be inferred that acceleration is inverse to the velocity. It forms a negative feedback effect that can make the DC voltage approach the equilibrium point. From the phenomenon, the motion of DC voltage is approaching the equilibrium point, which validates the analysis.

During from the instant $t_{2}$ to instant $t_{3}, \operatorname{RoCoV}$ is negative and dynamic deviation is negative as well, which suggests it forms a positive feedback, that the RoCoV can compel the dynamic deviations continuously changed. In this case, DC voltage cannot be stabilized at an equilibrium point because of positive feedback effect.

From instant $t_{3}$ to instant $t_{4}$, it forms a negative feedback effect, and the DC voltage is dynamically approaching the equilibrium point, and the dynamic deviation is gradually decreased to zero.

Discussion 2: The dynamic interaction between the RoCoV and DCVN is developed during the whole transient response from the instant of disturbance to the steady state (or to the divergent unstable state if instability). From the aforementioned analysis, the voltage waveform of transient response due to a mismatched/imbalanced power can coincide with the voltage waveform of oscillation due to current oscillation. Besides, two indices, i.e., RoCoV and DCVN are similar with the oscillation frequency and oscillation magnitude, respectively.

Hence, it is significant to identify the impact of RoCoV as well as DCVN on the dynamics of oscillation. Besides, the dynamic interaction between $\operatorname{RoCoV}$ and $\mathrm{DCVN}$ is also discussed here to clarify the motion of DC voltage with feedback effect. It can be inferred from Fig. 9(a) that the maximum deviation, i.e., DCVN and RoCoV are declined simultaneously over time, which indicates the system forms a negative feedback effect. From another view, both RoCoV and DCVN are gets zero when system reaches to an equilibrium point. RoCoV not only can reflect the variation speed of DC voltage, but also can reflect the extent of imbalanced current. Motion of the voltage tends to be stable when and only when both DCVN and RoCoV are equal to zero. This phenomenon is as the same as that of oscillation. Vice versa, both RoCoV and DCVN get larger and larger if the system forms a positive feedback effect.

For example, with the imbalanced fluctuation of current, voltage is naturally charged and climb to the peak value until DC capacitor begins to discharge. However, the owned inertia of system can compel the voltage maintain the original state and not to climb. Thus, DC voltage will climb slower with less deviation to the nominal. Similarly, inertia makes the voltage drop slower with less deviation in the descending process. Hence, the larger inertia can make the DC voltage slower and deviate less, as shown in Fig. 10(a). It can be seen that although size of inertia can impose effect on both RoCoV and DCVN, compared to DCVN, the impact of inertia on RoCoV is greater. Greater inertia leads to much slower RoCoV as well as less DCVN, which can make the DC voltage smoother.

Furthermore, damping contributes to impeding the further changes of DC voltage during the disturbance as shown in Fig. 10(b). With the motivation of periodical fluctuation of DC current, voltage across DC capacitors will be oscillated around the nominal because of periodical charging and discharging of the capacitors. However, the damping effect can hinder it to be further changed, and the attenuated energy makes it deviate less in the next cycle. The stronger damping can impede motion of DC voltage and thus deviates less.

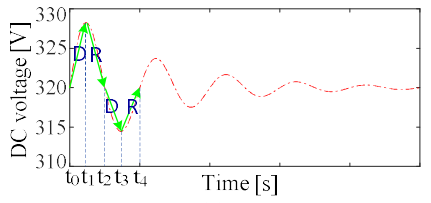

(a)

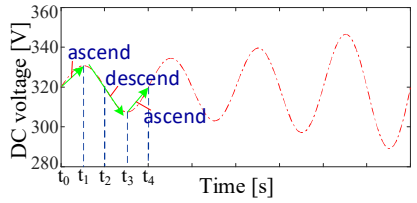

(b)
Fig. 9. Illustration of motion of DC voltage with feedback effect, (a) negative feedback effect, (b) positive feedback effect.

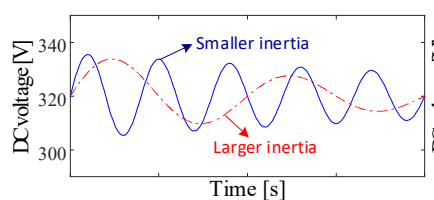

(a)

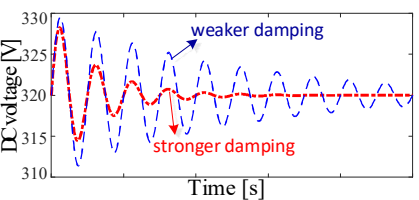

(b)
Fig. 10 The impact of inertia and damping on the dynamic response of DC voltage, (a) various levels of inertia, (b) various levels of damping



(a)

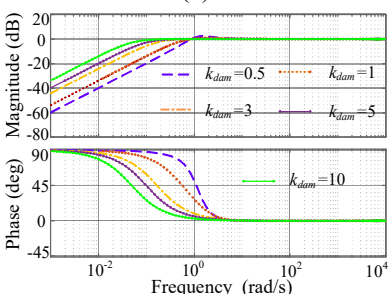

(c)

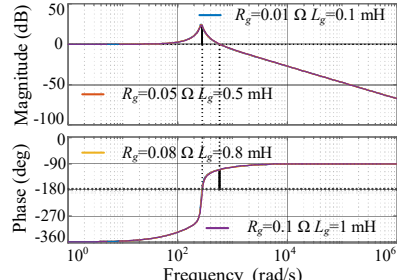

(b)

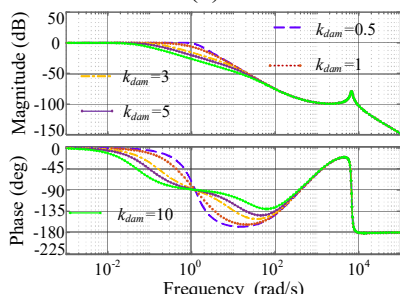

(d)
Fig. 11. Robustness performance and robust stability analysis, (a) Sensitivity function (defined as $S$ ) with conventional control (CC), (b) complementary sensitivity function (defined as $T$ ) with $\mathrm{CC}$, (c) $S$ with virtual synchronous control, (d) $T$ with virtual synchronous control.

Furthermore, the greater size of damping results in less DCVN and much slower RoCoV. Besides of this, the stronger damping could make the DC voltage oscillation attenuated much faster than that of weaker damping. Compared to weaker damping, stronger damping leads to the negative feedback effect, that RoCoV and DCVN gets smaller and smaller, more obvious. It means that stronger damping can bring more obvious negative feedback effect for the stabilization of the motion of voltage.

\section{Robustness performance analysis}

The sensitivity and complementary sensitivity are required to be defined as $S$ and $T$, expressed as: 


$$
\left\{\begin{array}{l}
T=\frac{L}{1+L} \\
S=\frac{1}{1+L}
\end{array}\right.
$$

For robustness performance analysis, it is required to define the peak value of $S$ and $T$ by means of $H_{\infty}$ norm, i.e.,

$$
\left\{\begin{array}{l}
N_{S}=\underbrace{\max }_{\omega}|S(j \omega)|=\|S\|_{\infty} \\
N_{T}=\underbrace{\max }_{\omega}|T(j \omega)|=\|T\|_{\infty}
\end{array}\right.
$$

Thus, to acquire the good enough robustness performance and robust stability margins, the infinite norm of both $S$ and $T$ are described as:

$$
\left\{\begin{array}{c}
\mathrm{RS} \Leftrightarrow\|T\|_{\infty} \leq 1 \\
\mathrm{RP} \Leftrightarrow\|S\|_{\infty}+\|T\|_{\infty} \leq 1
\end{array}\right.
$$

As shown in Fig. 11, both $S$ and $T$ are acquired to identify the robustness performance and robust stability. Fig. 11(a) and (b) depicts the frequency response of $S$ and $T$ with conventional dual loop control method. And the resonance peaks appear in both $S$ and $T$, which indicates poor robust stability and robustness performance.

As shown in Fig. 11(c) and (d), the resonance peaks are weakened by the proposed virtual synchronous control method, and the infinite norm of both $S$ and $T$ are below 1 (absolute value 1 means $0 \mathrm{~dB}$ ). Hence, the proposed virtual synchronous control can weaken the resonance peaks and enhance robustness performance and improve the robust stability margins.

\section{E. The energy source of inertia}

As can be seen in Fig. 5(b), the gain of current-loop $G_{\text {in }}$ can be seen as unit since the response in time scale of inductor-current is much faster than other loops, which leads to $\Delta I_{v i r}=\Delta I_{i n}$. That is, the virtual DC current is equal to output current of the converter, i.e., the controlled current. Indeed, the virtual synchronous controller will take actions to release the required energy to provide inertial support, when a disturbance appears which leads to the variation of DC voltage. According to the swing equation of virtual synchronous in (4), it can be known that the damping power contributes to enhancing damping performance meanwhile provide inertial support, i.e.,

$$
J_{v i r}^{\prime}=\frac{\frac{d \omega_{v i r}}{d t}}{k_{d a m}\left(\omega_{v i r}-\omega_{N}\right)}
$$

where the virtual inertia $J_{v i r}$ ' is equivalent to the obtained inertia by damping power, it can be also expressed as:

$$
J_{v i r}{ }^{\prime}=\frac{0.5 \omega_{v i r}{ }^{2}}{\int_{0}^{T_{s}} k_{d a m} \omega_{n}\left(\omega_{v i r}-\omega_{n}\right) d t}
$$

where it is derived based on energy conservation, and $T_{s}$ means the time that DC voltage reaches the new steady equilibrium point. Indeed, the supplementary of inertia and damping is supported by the built virtual DC voltage $V_{\text {vir }}$ and the obtained virtual current $I_{\text {vir }}$, which are the output of the virtual synchronous controller.
However, the energy of built virtual voltage is originated from the energy storage, which supplies the required energy to support inertia and damping. The dynamic response during support of inertia as well as damping can be described by the energy conservation equation, i.e.,

$$
\int_{0}^{T_{s}} \frac{V_{v i r}+V_{d c}}{k_{\text {virt }}} V_{d c} d t=\int_{0}^{T_{s}} I_{d c} V_{d c} d t+\int_{0}^{T_{s}} C V_{d c} \frac{d V_{d c}}{d t} d t
$$

Besides, the virtual current $I_{v i r}$ is desired to be equal to $I_{d c}$ in the steady equilibrium point. Thus, the larger $k_{\text {virt }}$ can make the built virtual voltage $V_{v i r}$ higher, which means that it can provide larger inertia support and more required energy. It can be inferred that $k_{\text {virt }}$ adds a control freedom degree for the system to regulate the damping and inertia for the DC microgrid.

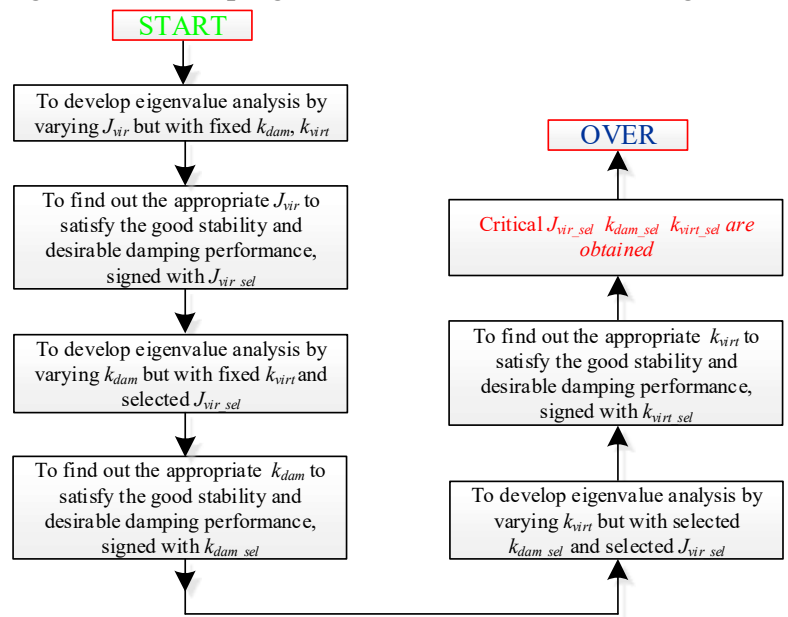

Fig. 12. The flow chart of tuning control parameters of the proposed virtual synchronous control method.

\section{F. Parameters selection}

The selection of system specifications is essential for the design of DC-DC converter which contains both the hardware part and control part.

Once the hardware parameters have been determined, it can be hardly changed. Thus, a good way is to select the circuit hardware parameters firstly as the control parameters are flexible to be controlled to satisfy the dynamic performance of the system. Hence, the parameters selection can be developed step by step as followings:

1) Control parameters selection of $J_{v i r}, k_{d a m}$, and $k_{\text {virt }}$

The final step of the controller parameters tuning is the selection of the presented control algorithm, i.e., selection of $J_{v i r}, k_{d a m}$, and $k_{\text {virt }}$. It should be noted that the premise is that the proportional integral parameters are fixed prior to it. Those can be determined from various perspectives, i.e.,

f1) Perspective of assurance of good stability

As the hardware parameters and control parameters of dual-loop have been determined in superior step, tuning of $J_{v i r}$, $k_{d a m}$, and $k_{\text {virt }}$ can be developed by the eigenvalues guidelines from perspective of assurance of good stability.

Fig. 12 displays the tuning procedure of the control parameters of virtual synchronous control strategy, e.g., tuning of $J_{v i r}, k_{\text {dam }}, k_{\text {virt }}$. To obtain the expected dynamic performance of the system, appropriate control parameters can be flexibly tuned by procedure in Fig. 12. 
The first step is to develop eigenvalue analysis by varying $J_{v i r}$ but with fixed $k_{d a m}$ and $k_{v i r t}$. Afterwards, an optimum $J_{v i r}$ can be found out to satisfy the good stability and desirable dynamic performance, signed with the selected $J_{v i r}$ sel . Then, the second step aims to develop eigenvalue analysis by varying $k_{d a m}$ but with fixed $k_{\text {virt }}$ and the selected $J_{\text {vir sel }}$. Afterwards, the optimum value of $k_{d a m}$, signed with $k_{\text {dam sel }}$, can be found out by identifying the desirable damping performance region. Finally, the third step is to carry out the eigenvalue analysis by varying $k_{\text {virt }}$ to find out the optimum $k_{\text {virt sel }}$. And the detailed procedure can be seen in Fig. 12. For this operation, the optimum tuning can be realized for the three control parameters.

f2) Perspective of transient inertial support

As shown in Fig. 5, the virtual synchronous (VS) DC current $I_{v i r}$ is the controlled current of DC converter, which is justly equal to the output current, when system reaches to an equilibrium point. It can be seen in Fig. 5(b), the small perturbation increment of the virtual DC voltage $\Delta V_{v i r}$ can be used for the inertial support and thus generate the required current $\Delta I_{v i r}$ for inertial support.

It can be seen in Fig. 8(c) that $k_{\text {virt }}$ cannot be selected too small to guarantee the positive damping of the dominant poles. In addition, the greater $J_{v i r}$ can lead to less dynamic voltage deviations and less oscillation magnitude of the voltage. Besides, the greater $J_{v i r}$ can also decease the settling time of the voltage which returns back to the steady state point much faster.

Fig. 6(b) indicates the greater $k_{d a m}$ results in less restoration time and less fluctuation range of the voltage, and the better damping and inertial support can be provided.

Actually, the tuning should take the remaining capacity of the power converter which can realize the inertial support into account. Hence, the control parameters tuning should be considered comprehensively with the discussed indices aforementioned to satisfy the superior performance of the system.

\section{Simulations AND EXPERIMENTS}

\section{A. Simulations}

In this Section, simulations and experiments are developed to demonstrate the proposed ideas based on a setup of a DC microgrid with three DC boost converters, which is connected to DC bus via DC line with feeding DC loads. Table II shows the specifications of the systems in the simulations.

Fig. 13 displays the waveforms of DC voltage across DC capacitor and DC current through DC lines, respectively. It can be seen the voltage and current oscillation is induced, but is dampened when virtual synchronous control is put into operation.

Fig. 14 depicts the transient IRs when the DC microgrid is disturbed in a form of load increase. The units of measurement of the control parameters, e.g., $J_{\text {vir }}, k_{\text {dam }}, k_{\text {virt }}$, are The impact of various size of virtual damping $k_{d a m}$ on the transient response of DC voltage is discussed. It can be seen in Fig. 14(b) that larger virtual damping leads to smaller dynamic deviation of DC voltage, i.e., smaller DCVN.

It can be seen in Fig. 14(c) that the voltage oscillation occurs when $k_{v i r t}=10$, which illustrates that small enough $k_{v i r t}$ can provide insufficient damping for DC microgrid and causes the voltage oscillation. As $k_{\text {virt }}$ increases, the voltage oscillation disappears, which indicates that the larger size of $k_{\text {virt }}$ can bring stronger damping for the system.

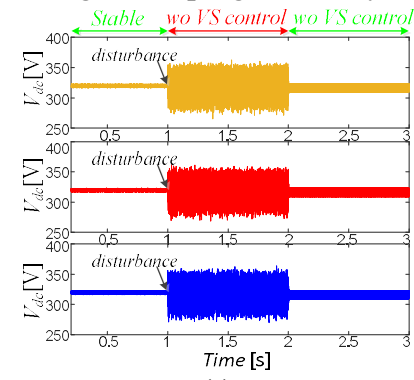

(a)

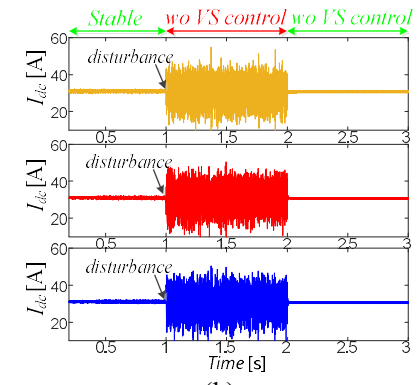

(b)
Fig. 13. Simulation results of DC voltage oscillation and oscillation damping by VSG control.

TABLE II. SySTEM SPECIFICATIONS OF SIMULATIONS

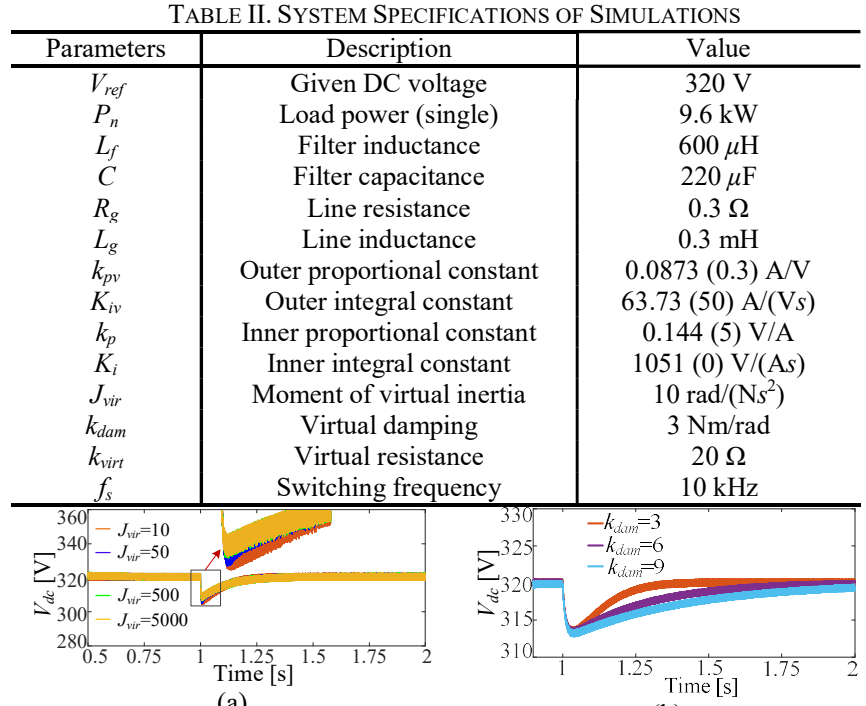

(a)

(b)

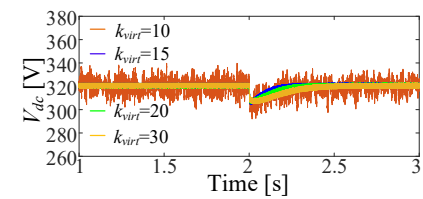

(c)

Fig. 14. The impact of virtual synchronous control parameters on the DCVN and RoCoV, (a) changing $J_{v i r}$, (b) changing $k_{d a m}$, (c) varying $k_{\text {virt }}$.

\section{B. Experiments}

Figs. 15 and 16 show the Star-Sim HIL results of DC voltages and currents. It should be noted that DCVO and DCCO means the oscillation of DC voltage and DC current. DCVS means virtual synchronous control put into operation.

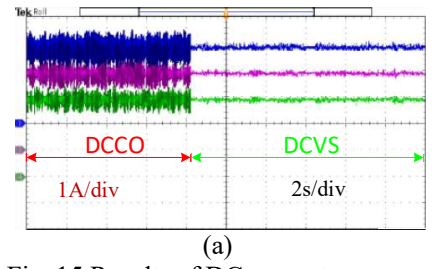

Fig. 15 Results of DC current

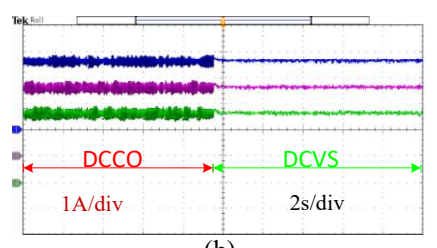

(b)
It can be seen that the system is prone to oscillation due to the weak damping of the system. However, the proposed virtual synchronous control can effectively dampen the oscillation and 
maintain the synchronization operation. Moreover, the current quality is improved as well.

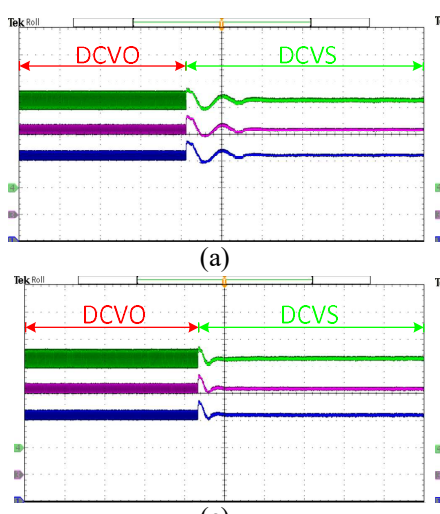

(c)

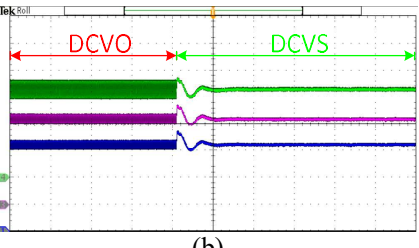

(b)

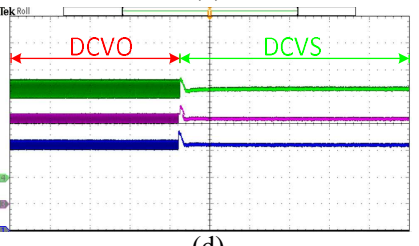

(d)
Fig. 16 The impact of virtual inertia on voltage oscillation damping and RoCoV, (a) $J_{v i r}=0.5$, (b) $J_{v i r}=1$, (c) $J_{v i r}=2$, (d) $J_{v i r}=5$.

TABLE III COMPARISON PERFORMANCE INDICATORS BETWEEN VIRTUAL SYNCHRONOUS CONTROL AND CONVENTIONAL DUAL-LOOP CONTROL

\begin{tabular}{c|c|c|c|c}
\hline PI & $\begin{array}{c}\text { RoCoV } \\
(\max )\end{array}$ & $\begin{array}{c}\text { DCVN } \\
(\max )\end{array}$ & VOF & VOA \\
\hline $\begin{array}{c}\text { Conventional dual } \\
\text { loop control }\end{array}$ & $226 \mathrm{~V} / \mathrm{s}$ & $5 \mathrm{~V}$ & $226 \mathrm{~Hz}$ & $5 \mathrm{~V}$ \\
$\begin{array}{c}\text { Proposed virtual } \\
\text { synchronous } \\
\text { control }\left(J_{\text {vir }}=0.5\right)\end{array}$ & $\begin{array}{c}0.5 \mathrm{~V} / \mathrm{s} \\
\text { (attenuated } \\
\text { to } 0 \mathrm{~V} / \mathrm{s})\end{array}$ & $\begin{array}{c}5 \mathrm{~V} \\
\text { (attenuated } \\
\text { to } 0 \mathrm{~V})\end{array}$ & $\begin{array}{c}0.5 \mathrm{~Hz} \\
\text { (gradually } \\
\text { be stable) }\end{array}$ & $\begin{array}{c}5 \mathrm{~V} \\
\text { (finally } \\
\text { to be } 0 \\
\mathrm{~V})\end{array}$ \\
\hline
\end{tabular}

Table III presents the comparison performance indicators (PI) between virtual synchronous control and dual-loop control. It is found that the RoCoV and maximum DCVN are deceased after the virtual synchronous control introduction. The PI of RoCoV is closely related VOF, and maximum DCVN corresponds to VOA. The unified concept is developed between the inertial response and ORS. Actually, the current oscillation means the imbalanced current through DC capacitors which could charge or discharge the capacitors. It should be noted that there still remains voltage ripples caused by the switching actions of the transistors, which is not the focused problem in this paper. It should be noted that all of the performance indicators in this paper refers to the stability issues caused by imbalanced powers, weak damping, or undesirable interactions, which does not include the type of ripples caused by switching actions. Thus, that DCVN and VOA tend to be zero refers to the oscillation magnitude or voltage nadir by small signal average model. It is not applicable for the ripples originated from switching actions. All of the analysis is based on the small signal average model, which does not include the high-frequency ripples by switching actions.

The power stage of d-SPACE controller based hardware experimental setups comprised DC power supply, boost converters, L-filters, DC capacitors, and power loads. The algorithm was implemented in the dSpace MicroLabBox with DS1202 PowerPC DualCore $2 \mathrm{GHz}$ processor board. The simulations need to be tuned well enough as the same parameters as experiments before conducting d-Space controller hardware experiments. During experiments, an ENABLE should be set in the control algorithm to make all of the integral control not action before the hardware is put into operation. It means that before the DC power supply is put on and hardware gets into operation, the ENABLE button should be set off (ENABLE $=0)$. That operation aims to guarantee the normal startup of the system and effective action of the control algorithm. Table IV shows the experiments specification, and hardware parameters for each converter are the same.

To further test the synchronizing tracking capability of the proposed control algorithm, experiments are developed based on a three-converters DC microgrids. It should be noted that the synchronization of DC voltage means the real voltage can track well with the given reference value (nominal voltage). DC microgrids is constituted into three paralleled DC boost converters which feeding the power loads.

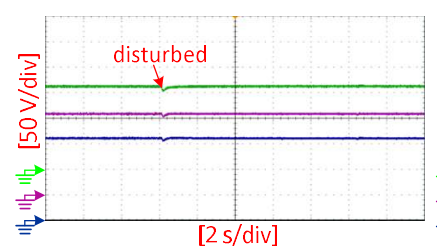

(a)

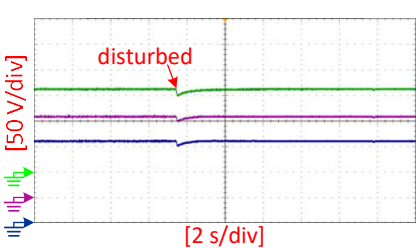

(b)
Fig. 18 d-Space experimental results for test of synchronized tracking performance of the control algorithm, (a) case 1, (b) case 2

TABLE IV. SYSTEM SPECIFICATIONS OF EXPERIMENTS

\begin{tabular}{c|c|c}
\hline Parameters & Description & Value \\
\hline$V_{d c}$ & DC operation voltage & $160 \mathrm{~V}$ \\
$R_{L}$ & Load resistance & $57.5 \mathrm{ohm}($ adjustable) \\
$R_{t}$ & Total load resistance & $57.5 / 3$ \\
$L_{f}$ & Filter inductance & $0.8 \mathrm{mH}$ \\
$C$ & Filter capacitance & $1100 \mu \mathrm{F}(2200 / 2)$ \\
$N_{R}$ & Amount of paralleled $R_{L}$ & 3 \\
$N_{L}$ & Total amount of L-filter & 3 \\
$N_{C}$ & Total amount of DC capacitors & 6 \\
$N_{d c \text { boost }}$ & Amount of DC boost converters & 3 \\
$J_{v i r}$ & Moment of virtual inertia & adjustable $\left(\mathrm{rad} /\left(\mathrm{Ns} s^{2}\right)\right)$ \\
$k_{d a m}$ & Virtual damping & adjustable $(\mathrm{Nm} / \mathrm{rad})$ \\
$k_{\text {virt }}$ & Virtual resistance & adjustable $(\Omega)$ \\
$f_{\text {swi }}$ & Switching frequency & $5 \mathrm{kHz}$ \\
\hline
\end{tabular}

Fig. 18 shows the d-Space experimental results. This case of experiment is to validate the synchronization performance of the control algorithm. Thus, no switches of the control modes are considered in the cases. It can be seen that a small voltage dip occurs after the disturbance, and recovers to the nominal value with a short transients without any oscillation. It shows a good synchronization performance of the system with good damping performance.

\section{CONCLUSIONS AND DisCUSSIONS}

This paper proposes a virtual synchronous control strategy to maintain the synchronization operation of DC converter with grid. Besides, it can improve the damping performance, inertia, RoCoV, DCVN, and robustness as the control parameters is flexible to be settled.

This paper relates the RoCoV and DCVN, which usually appears in the IR, with the ORS for the first time. A unified concept is put forward for relating the RoCoV and DCVN with the ORS. Especially, a comparison of performance indicators among RoCoV, DCVN, voltage oscillation frequency, and voltage oscillation magnitude is developed. Through theoretical analysis, it is found that transient response of DC voltage due to imbalanced currents or mismatched powers is 
essentially the voltage oscillation which is originated from periodical fluctuation of DC current.

The dynamic interaction between RoCoV and DCVN is discussed with the feedback effect. It can be concluded that both RoCoV and DCVN gets smaller and smaller thereby finally tends to be zero when the system forms a negative feedback effect. While both RoCoV and DCVN gets greater and greater thereby finally tends to be infinite when the system forms a positive feedback effect. The RoCoV and DCVN is related with ORS through feedback effect, and those can be recognized as indicators of motion of voltage.

The inertia can be recognized as another type of damping to impede the further change of the state variables and maintain close to the original state for synchronization operation. Thus, the inertia plays a similar role as the damping.

Finally, quantification analysis between the two most important indices, RoCoV and DCVN, as well as the impact on the motion of DC voltage will be studied in our future works in a DC microgrid.

\section{REFERENCES}

[1] F. Blaabjerg and K. Ma, "Future on Power Electronics for Wind Turbine Systems," in IEEE Journal of Emerging and Selected Topics in Power Electronics, vol. 1, no. 3, pp. 139-152, Sept. 2013.

[2] G. Pinares and M. Bongiorno, "Analysis and Mitigation of Instabilities Originated From DC-Side Resonances in VSC-HVDC Systems," in IEEE Transactions on Industry Applications, vol. 52, no. 4, pp. 2807-2815, July-Aug. 2016

[3] M. Hadjikypris, V. Efthymiou and G. E. Georghiou, "Enhanced Frequency Response of Inverter Dominated Low Inertia Power Systems," 2019 1st International Conference on Energy Transition in the Mediterranean Area (SyNERGY MED), Cagliari, Italy, 2019, pp. 1-6.

[4] N. Rashidirad, M. Hamzeh, K. Sheshyekani and E. Afjei, "High-Frequency Oscillations and Their Leading Causes in DC Microgrids," in IEEE Transactions on Energy Conversion, vol. 32, no. 4, pp. 1479-1491, Dec. 2017

[5] M. N. Hussain, R. Mishra and V. Agarwal, "A Frequency-Dependent Virtual Impedance for Voltage-Regulating Converters Feeding Constant Power Loads in a DC Microgrid," in IEEE Transactions on Industry Applications, vol. 54, no. 6, pp. 5630-5639, Nov.-Dec. 2018.

[6] L. Guo, S. Zhang, X. Li, Y. W. Li, C. Wang and Y. Feng, "Stability Analysis and Damping Enhancement Based on Frequency-Dependent Virtual Impedance for DC Microgrids," in IEEE Journal of Emerging and Selected Topics in Power Electronics, vol. 5, no. 1, pp. 338-350, March 2017.

[7] Z. Wang, F. Zhuo, H. Yi, J. Wu, F. Wang and Z. Zeng, "Analysis of Dynamic Frequency Performance Among Voltage-Controlled Inverters Considering Virtual Inertia Interaction in Microgrid," in IEEE Transactions on Industry Applications, vol. 55, no. 4, pp. 4135-4144, July-Aug. 2019

[8] J. Liu, Y. Miura, H. Bevrani and T. Ise, "Enhanced Virtual Synchronous Generator Control for Parallel Inverters in Microgrids," in IEEE Transactions on Smart Grid, vol. 8, no. 5, pp. 2268-2277, Sept. 2017

[9] K. Sakimoto, Y. Miura and T. Ise, "Stabilization of a power system with a distributed generator by a Virtual Synchronous Generator function," 8th International Conference on Power Electronics - ECCE Asia, Jeju, 2011, pp. 1498-1505.

[10] J. Alipoor, Y. Miura and T. Ise, "Power System Stabilization Using Virtual Synchronous Generator With Alternating Moment of Inertia," in IEEE Journal of Emerging and Selected Topics in Power Electronics, vol. 3, no. 2, pp. 451-458, June 2015.

[11] Y. Hirase, K. Abe, O. Noro, K. Sugimoto and K. Sakimoto, "Stabilization effect of virtual synchronous generators in microgrids with highly penetrated renewable energies," 2016 IEEE 17th Workshop on Control and Modeling for Power Electronics (COMPEL), Trondheim, 2016, pp. $1-8$

[12] Y. Hirase, K. Sugimoto, K. Sakimoto and T. Ise, "Analysis of Resonance in Microgrids and Effects of System Frequency Stabilization Using a
Virtual Synchronous Generator," in IEEE Journal of Emerging and Selected Topics in Power Electronics, vol. 4, no. 4, pp. 1287-1298, Dec. 2016.

[13] K. Visscher and S. W. H. De Haan, "Virtual synchronous machines (VSG' s) for frequency stabilisation in future grids with a significant share of decentralized generation," CIRED Seminar 2008: SmartGrids for Distribution, Frankfurt, 2008, pp. 1-4.

[14] C. Li, Y. Li, Y. Cao, H. Zhu, C. Rehtanz and U. Häger, "Virtual Synchronous Generator Control for Damping DC-Side Resonance of VSC-MTDC System," in IEEE Journal of Emerging and Selected Topics in Power Electronics, vol. 6, no. 3, pp. 1054-1064, Sept. 2018.

[15] Y. Yang, C. Li, J. Xu, F. Blaabjerg and T. Dragicevic, "Virtual Inertia Control Strategy for Improving Damping Performance of DC Microgrid with Negative Feedback Effect," in IEEE Journal of Emerging and Selected Topics in Power Electronics. doi: 10.1109/JESTPE.2020.2998812

[16] X. Zhu, F. Meng, Z. Xie and Y. Yue, "An Inertia and Damping Control Method of DC-DC Converter in DC Microgrids," in IEEE Transactions on Energy Conversion, vol. 35, no. 2, pp. 799-807, June 2020.

[17] N. Zhi, K. Ding, L. Du and H. Zhang, "An SOC-based Virtual DC Machine Control for Distributed Storage Systems in DC Microgrids," in IEEE Transactions on Energy Conversion.

[18] J. Chen and T. O'Donnell, "Parameter Constraints for Virtual Synchronous Generator Considering Stability," in IEEE Transactions on Power Systems, vol. 34, no. 3, pp. 2479-2481, May 2019.

[19] J. Khazaei, Z. Tu and W. Liu, "Small-Signal Modeling and Analysis of Virtual Inertia-Based PV Systems," in IEEE Transactions on Energy Conversion, vol. 35, no. 2, pp. 1129-1138, June 2020.

[20] H. Wu et al., "Small-Signal Modeling and Parameters Design for Virtual Synchronous Generators," in IEEE Transactions on Industrial Electronics, vol. 63, no. 7, pp. 4292-4303, July 2016.

[21] J. Alipoor, Y. Miura and T. Ise, "Stability Assessment and Optimization Methods for Microgrid With Multiple VSG Units," in IEEE Transactions on Smart Grid, vol. 9, no. 2, pp. 1462-1471, March 2018.

[22] N. Soni, S. Doolla and M. C. Chandorkar, "Inertia Design Methods for Islanded Microgrids Having Static and Rotating Energy Sources," in IEEE Transactions on Industry Applications, vol. 52, no. 6, pp. 5165-5174, Nov.-Dec. 2016.

[23] J. Fang, P. Lin, H. Li, Y. Yang and Y. Tang, "An Improved Virtual Inertia Control for Three-Phase Voltage Source Converters Connected to a Weak Grid," in IEEE Transactions on Power Electronics, vol. 34, no. 9, pp. 8660-8670, Sept. 2019.

[24] J. Fang, Y. Tang, H. Li and X. Li, "A Battery/Ultracapacitor Hybrid Energy Storage System for Implementing the Power Management of Virtual Synchronous Generators," in IEEE Transactions on Power Electronics, vol. 33, no. 4, pp. 2820-2824, April 2018.

[25] C. Li, Y. Yang, Y. Cao, L. Wang, T. Dragicevic and F. Blaabjerg, "Frequency and Voltage Stability Analysis of Grid-forming Virtual Synchronous Generator Attached to Weak Grid," in IEEE Journal of Emerging and Selected Topics in Power Electronics. doi: 10.1109/JESTPE.2020.3041698

[26] A. Riccobono and E. Santi, "Comprehensive Review of Stability Criteria for DC Power Distribution Systems," in IEEE Transactions on Industry Applications, vol. 50, no. 5, pp. 3525-3535, Sept.-Oct. 2014.

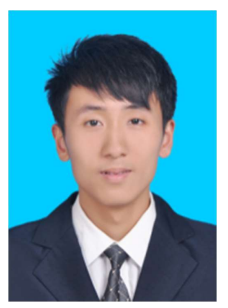

Chang Li received the Bachelor degree and the Ph.D. degree in Electrical Engineering from the Guizhou University and Hunan University in 2014 and 2020, respectively.

His current research interests include: stabilization and control of grid-forming inverters with grid-integration of renewable energy, Dynamics analysis of renewable energy generators, synthetic inertia control applied in DC microgrids.

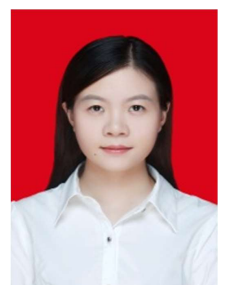

Yaqian Yang received the Master degree in Electrical Engineering from the Changsha University of Science and Technology in 2018. And she is pursuing P.h.D degree in Hunan University since 2018.

Her current research interests include: stabilization and control of grid-forming virtual synchronous generators, synthetic inertial control applied in hybrid AC/DC microgrids. 


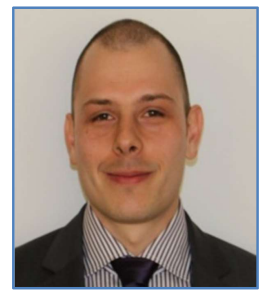

Tomislav Dragičević (S'09-M'13-SM'17) received the M.Sc. and the industrial Ph.D. degrees in Electrical Engineering from the Faculty of Electrical Engineering, University of Zagreb, Croatia, in 2009 and 2013, respectively. From 2013 until 2016 he has been a Postdoctoral researcher at Aalborg University, Denmark. From 2016 until 2020 he was an Associate Professor at Aalborg University, Denmark. Currently, he is a Professor at the Technical University of Denmark.

He made a guest professor stay at Nottingham University, UK during spring/summer of 2018. His research interest is application of advanced control, optimization and artificial intelligence inspired techniques to provide innovative and effective solutions to emerging challenges in design, control and diagnostics of power electronics intensive electrical distributions systems and microgrids. He has authored and co-authored more than 300 technical publications (more than 150 of them are published in international journals, mostly in IEEE), 10 book chapters and a book in the field.

$\mathrm{He}$ serves as an Associate Editor in the IEEE TRANSACTIONS ON INDUSTRIAL ELECTRONICS, in IEEE TRANSACTIONS ON POWER ELECTRONICS, in IEEE Emerging and Selected Topics in Power Electronics and in IEEE Industrial Electronics Magazine. Dr. Dragičević is a recipient of the Končar prize for the best industrial PhD thesis in Croatia, a Robert Mayer Energy Conservation award, and he is a winner of an Alexander von Humboldt fellowship for experienced researchers.

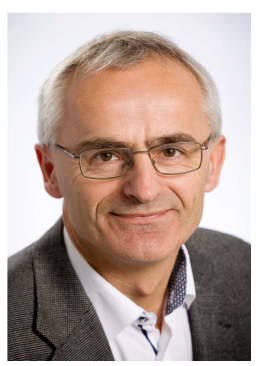

Frede Blaabjerg (S'86-M'88-SM'97-F'03) was with ABB-Scandia, Randers, Denmark, from 1987 to 1988. From 1988 to 1992 , he got the PhD degree in Electrical Engineering at Aalborg University in 1995. He became an Assistant Professor in 1992, an Associate Professor in 1996, and a Full Professor of power electronics and drives in 1998. From 2017 he became a Villum Investigator. He is honoris causa at University Politehnica Timisoara (UPT), Romania and Tallinn Technical University (TTU) in Estonia.

His current research interests include power electronics and its applications such as in wind turbines, PV systems, reliability, harmonics and adjustable speed drives. He has published more than 600 journal papers in the fields of power electronics and its applications. He is the co-author of four monographs and editor of ten books in power electronics and its applications.

He has received 33 IEEE Prize Paper Awards, the IEEE PELS Distinguished Service Award in 2009, the EPE-PEMC Council Award in 2010, the IEEE William E. Newell Power Electronics Award 2014, the Villum Kann Rasmussen Research Award 2014, the Global Energy Prize in 2019 and the 2020 IEEE Edison Medal. He was the Editor-in-Chief of the IEEE TRANSACTIONS ON POWER ELECTRONICS from 2006 to 2012. He has been Distinguished Lecturer for the IEEE Power Electronics Society from 2005 to 2007 and for the IEEE Industry Applications Society from 2010 to 2011 as well as 2017 to 2018. In 2019-2020 he served as a President of IEEE Power Electronics Society. He has been Vice-President of the Danish Academy of Technical Sciences.

He is nominated in $2014-2020$ by Thomson Reuters to be between the most 250 cited researchers in Engineering in the world. 